\title{
Polarization sensitivity of optical resonant dipole antennas
}

\author{
Holger Fischer \\ holger.fischer@epfl.ch
}

Olivier J. F. Martin

\begin{abstract}
Swiss Federal Institute of Technology Lausanne (EPFL), Nanophotonics and Metrology Laboratory, 1015 Lausanne, Switzerland

Swiss Federal Institute of Technology Lausanne (EPFL), Nanophotonics and Metrology Laboratory, 1015 Lausanne, Switzerland
\end{abstract}

The polarization sensitivity of optical resonant dipole antennas is investigated numerically using the Green's tensor technique. The electric field-intensity in the feed-gap of the antenna is calculated as function of the polarization of the incident field. A simple analytical model is proposed that matches the numerical data very well. While a very strong polarization sensitivity can be achieved for specific wavelengths, our results also indicate that there are situations where the antenna is not sensitive at all to the polarization. The role played by different plasmon resonances in the system is illustrated. [DOI: 10.2971/jeos.2008.08018]

Keywords: dipole antenna, polarization sensitivity, plasmon resonance, Green's tensor method

\section{INTRODUCTION}

Antennas represent probably the archetype of classical electromagnetic devices and their properties at radio frequencies have been studied in great detail [1]. Recently there has been growing interest to downscale electromagnetic antennas towards optical frequencies by using plasmon resonances. First attempts are the dipole antenna [2], the bowtie antenna [3, 4] or simply metallic nanoparticles with all kinds of shapes [5][9]. Recent publications show that the strong plasmon resonances and the subsequent very high local electromagnetic fields are extremely useful to amplify emission and excitation of dipole emitters [10]. It has been shown that plasmonic nanoparticles can significantly enhance molecular emission and decrease the molecular excited state lifetime [11]-[13]. Applications in semiconductor devices for outcoupling or beaming light are also under investigation [14]-[17].

For classical antennas, the polarization sensitivity represents a key parameter both in emission and reception modes [1]. At optical frequencies, the polarization sensitivity determines the interaction with dipolar emitters such as fluorescent molecules or quantum dots [11]. The objective of this paper is to study the polarization sensitivity of such nanoscopic antennas under farfield illumination. The results presented here are also valid when the antenna is combined with a dipolar source placed in its gap. In that case, the orientation of the dipole determines the polarization of the illumination field. The coupling mechanisms between such an emitter and the antenna are however beyond the scope of this paper.

\section{SYSTEM AND METHOD}

We consider dipole nanoantennas of two equally sized, closely spaced gold nanorods with a small gap (Figure 1), where strong fields are created at the antenna resonance [2]. The feed-gap of the antenna thus plays a crucial role as interface to access the antenna resonance in either reception or emission mode. In this paper we shall thus concentrate on the investigation of the field inside the feed-gap. The Green's tensor method is used to investigate numerically the polarization sensitivity of the nanoantenna [18]-[20]. The modeled dipole antenna has an arm length of $100 \mathrm{~nm}$, a thickness and width of $40 \mathrm{~nm}$ and a gap width of $30 \mathrm{~nm}$. The values for the real and imaginary parts of the dielectric function of gold used for the calculations are obtained from experimental data [21]. The dipole antenna is illuminated with a plane wave at $70^{\circ}$ incidence from the vertical axis, as indicated by the red arrow in Figure 1. The incident angle is chosen to fulfill the total internal reflection condition at the glass/air interface. The incident k-vector is perpendicular to the antenna's long axis. The polarization of the incident field is then turned in steps of $1^{\circ}$ from $\Theta=0^{\circ}$ (polarization perpendicular to the long antenna axis) to $\Theta=90^{\circ}$ (polarization parallel to the long antenna axis). For each polarization the relative field intensity (relative to the illumination intensity) is calculated in the middle of the feed-gap (red point in Figure 1).

Depending on the illumination wavelength and the incident polarization the antenna supports two different resonances along either its short axis or its long axis (see intensity maps in Figure 2). In the chosen observation point the long axis resonance is expected to be much stronger than the short axis resonance, since the depolarization field occurs where the electric field is normal to the metal surface [22]. The spectral positions of these two resonances are visible in Figure 2, which shows the relative electric field intensity $I(\Theta, \lambda)$ in the middle of the gap as a function of the incident polarization angle $\Theta$ and the illumination wavelength $\lambda$. Note that a logarithmic scale is used for the intensity-axis of this figure. The long 


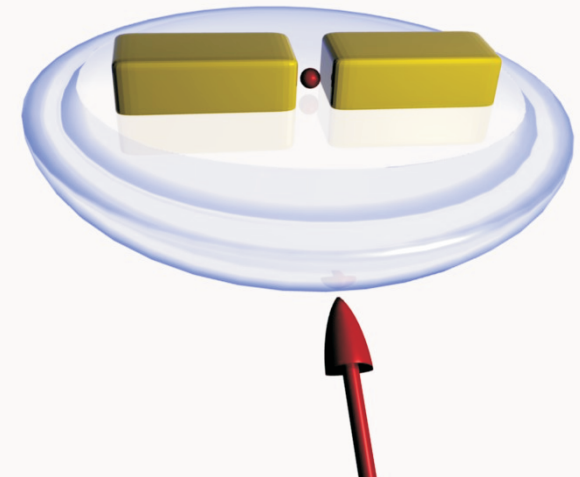

FIC. 1 A dipole gold nanoantenna on a glass substrate. The antenna is illuminated at an incident angle of $70^{\circ}$ (red arrow). The electric field intensity is calculated at the red point in the antenna feed-gap

axis resonance can be clearly identified at about $\lambda_{l}=760 \mathrm{~nm}$ indicated with a blue arrow. The corresponding relative field intensity map on a parallel plane $20 \mathrm{~nm}$ above the structure is also shown (Figure 2). The field enhancement between the antenna arms is different to that of the spectral plot, because of the different distance from the antenna. The short axis resonance is observed at about $\lambda_{s}=520 \mathrm{~nm}$ for small polarization angles and is indicated with a red arrow. The corresponding field intensity map above the antenna is also shown in this figure. It indicates a much lower field enhancement. Furthermore, the field maxima are now located rather at the edges of the structure and the intensity in the gap is low.

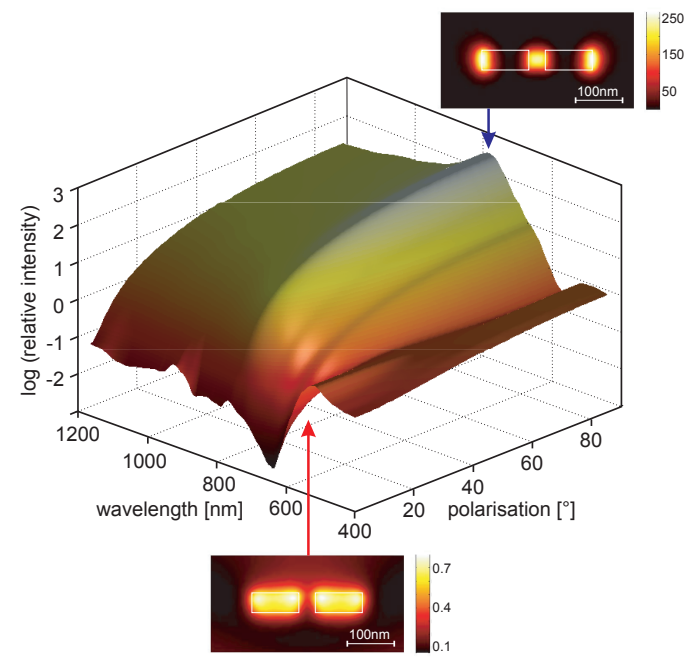

FIG. 2 Relative intensity in the feed-gap as a function of the incident wavelength and polarization angle. The red and the blue arrows indicate the position of the short and long axis resonances, where the corresponding electric field intensities are plotted on a plane $20 \mathrm{~nm}$ above the structure.

\section{DISCUSSION}

The field intensity in the feed-gap $I(\Theta, \lambda)$ for the long axis resonance $\lambda_{l}=760 \mathrm{~nm}$ depends on the incident field component along this antenna axis. Hence, the intensity should follow a simple square sinusoidal law with respect to the polarization angle:

$$
I(\Theta, \lambda)=I\left(90^{\circ}, \lambda\right) \sin ^{2} \Theta
$$

where $I\left(90^{\circ}, \lambda\right)$ is the intensity in the field gap, obtained from the numerical data, for light polarized purely parallel to the antenna axis $\left(\Theta=90^{\circ}\right)$.

At $\lambda_{l}=760 \mathrm{~nm}$ Eq. (1) describes the numerical data very well, as illustrated in Figure 3a. This figure shows the numerical (red crosses) and model results (blue line) for the relative intensity as a function of the polarization angle. Let us now investigate the validity of this simple model out of the main resonance. Figure $3 \mathrm{~b}$ shows the relative error function:

$$
\varepsilon(\Theta, \lambda)=\frac{\left|I_{\text {numerical }}(\Theta, \lambda)-I_{\text {model }}(\Theta, \lambda)\right|}{I_{\text {numerical }}(\Theta, \lambda)},
$$

for the intensity data $I_{\text {model }}$ obtained with Eq. (1) and the numerical data $I_{\text {numerical }}$ obtained by solving the complete scattering problem. The antenna spectrum at a polarization angle of $\Theta=4^{\circ}$, where the spectral positions of both resonances are clearly visible, is plotted as a black line in Figure 3b. Eq. (1) fits the numerical data very well for wavelengths longer than the long axis resonance and for large polarization angles. On the other hand, approaching the short axis resonance and small polarization angles, a large discrepancy is observed between model and numerical data. Apparently the short axis resonance plays a crucial role for the field intensity inside the gap and cannot be neglected for a complete description of the antenna polarization sensitivity at these wavelengths and polarization angles. We thus add the antenna short axis resonance to our model by simply introducing a square cosine function with the appropriate weighting $I\left(0^{\circ}, \lambda\right)$ obtained from the numerical data:

$$
I(\Theta, \lambda)=I\left(90^{\circ}, \lambda\right) \sin ^{2} \Theta+I\left(0^{\circ}, \lambda\right) \cos ^{2} \Theta .
$$

Figure $3 c$ shows the resulting relative error using Eq. (3). Again the antenna spectrum $I\left(4^{\circ}, \lambda\right)$ is added as black line to the density plot. Note the different scales for the density plots in Figures $3 b$ and c. The agreement between Eq. (3) and the numerical data is excellent, the maximum relative error being in the order of $10^{-5}$.

For the particular geometry of our antenna, the expression of Eq. (3) can be easily induced from symmetry arguments and its validity is confirmed by the numerical checks that were carried out. We want to emphasize that comparable expressions could be found for more general structures and geometries of the incident field. Once such a dependence on angle of incidence and state of polarization has been established, a strong gain in numerical efficiency is obtained.

Once the response of an antenna is known for two orthogonal polarization states, this analytical model provides its response for any required arbitrary polarization without intensive computational effort, whereas a full wave simulation for a single 
(a)

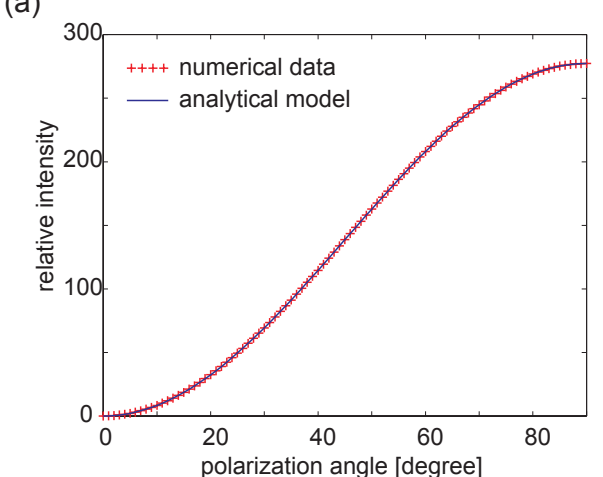

(b)

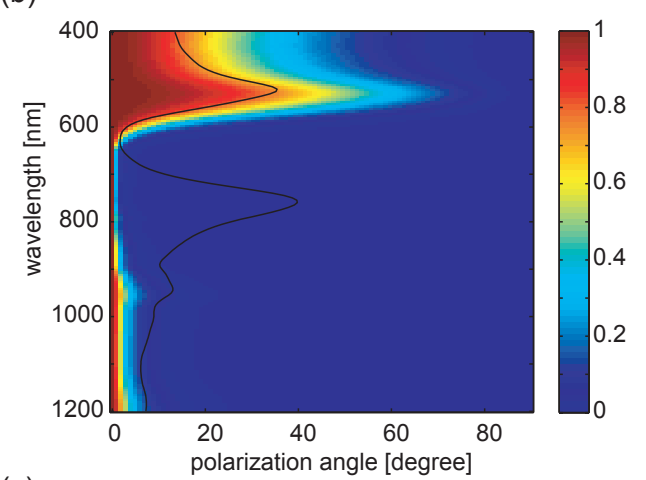

(c)

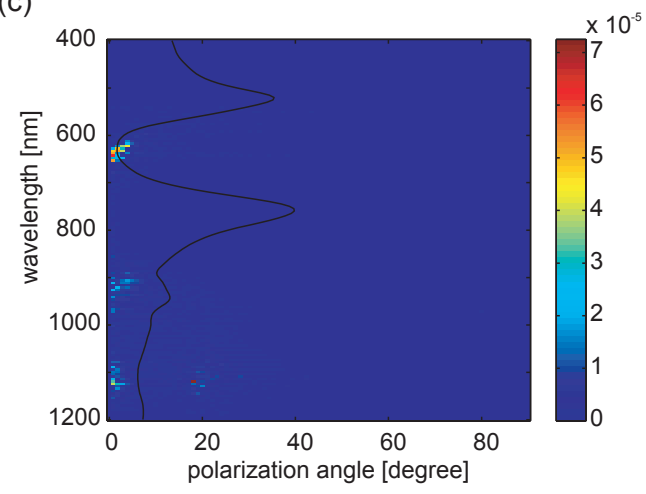

FIG. 3 Comparison of the two mathematical models with the numerical results: (a) At resonance for a purely square sinusoidal model, (b) error for a purely square sinusoidal model given by Eq. (1), and (c) error for a more complete model given by Eq. (3). Note the different color scales in (b) and (c). The solid black lines in (b) and (c) are the spectra at an incident polarization of $4^{\circ}$.

polarization can be quite computing intensive, even for the simplest structure.

If we define the polarization sensitivity as,

$$
s(\lambda)=10 \log _{10}\left(\left|I\left(90^{\circ}, \lambda\right) / I\left(0^{\circ}, \lambda\right)-1\right|\right),
$$

the polarization sensitivity should vanish at wavelengths where both resonances produce the same intensity contribution $\left(I\left(90^{\circ}, \lambda\right)=I\left(0^{\circ}, \lambda\right)\right)$. Note however that in this case the electric fields at these wavelengths are in general different, only their amplitudes and thus their intensities are the same.

Figure 4 shows the polarization sensitivity as a function of the incident wavelength. For a better understanding the antenna resonance spectra $10 \log _{10}\left(I\left(90^{\circ}, \lambda\right)\right)$ and $10 \log _{10}\left(I\left(0^{\circ}, \lambda\right)\right)$ are plotted as dotted lines. For wavelengths around the long axis resonance the sensitivity is maximal and reaches about $40 \mathrm{~dB}$, i.e. the intensity ratio perpendicular/parallel is about 1 : 10000. As predicted, around the short axis resonance there are two wavelengths where the sensitivity vanishes, and the intensity in the feed-gap remains the same for any arbitrary polarization state of the incident illumination. These two wavelengths $\left(\lambda_{1}=510 \mathrm{~nm}\right.$ and $\left.\lambda_{2}=545 \mathrm{~nm}\right)$ are marked in Figure 4 by two vertical lines. They are located at the intersections of the purely long axis and the purely short axis resonance spectra (dotted lines in Figure 4). Depending on the excitation wavelength a dipole antenna can thus be strongly polarization sensitive or completely polarization insensitive. To the best of our knowledge this is a unique feature of plasmonic dipole antennas which cannot be observed for classical dipole antennas working at radio frequencies.

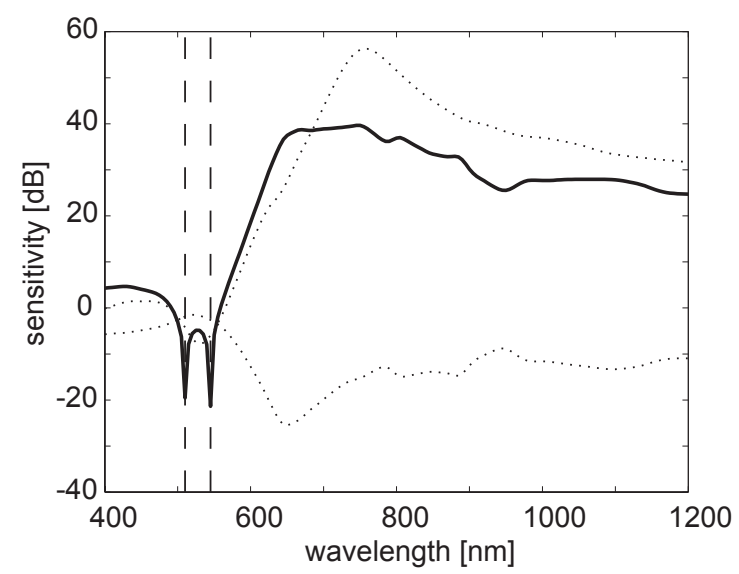

FIG. 4 Polarization sensitivity of the dipole antenna. The spectral positions where the sensitivity is zero are marked with the vertical lines. The dotted lines correspond to the intensities at $0^{\circ}$ and $90^{\circ}$.

\section{CONCLUSION}

The polarization sensitivity of an optical resonant metallic nanoantenna has been investigated. The interplay of the two plasmon resonances of such a structure dictates the antenna response on the polarization of the incident field. In particular, using a simple analytical model, it has been shown that both the short and the long axes plasmon resonances determine the intensity in the feed-gap. For wavelengths close to the long axis plasmon resonance, the antenna is strongly polarization sensitive (about $40 \mathrm{~dB}$ ), but as a consequence of the overlap of the two resonances there are spectral positions where the antenna is completely polarization insensitive. We believe that this study can help design more complicated structures with multiple resonances to tailor their polarization sensitivity spectrum. In that case, the modal decomposition of the structure's response provides a simple way to analyze the polarization sensitivity even for large and complex structures. 


\section{ACKNOWLEDGEMENTS}

Funding from the Swiss National Science Foundation (contracts 200020-113458 and R'Equip 206021) is gratefully acknowledged.

\section{References}

[1] C. A. Balanis, Antenna Theory Analysis and Design (John Wiley and Sons, inc., New York, 1997).

[2] P. Muhlschlegel, H. J. Eisler, 0. J. F. Martin, B. Hecht and D. W. Pohl, "Resonant Optical Antennas" Science 308, 1607-9 (2005).

[3] P. J. Schuck, D. P. Fromm, A. Sundaramurthy, G. S. Kino and W. E. Moerner, "Improving the Mismatch between Light and Nanoscale 0bjects with Gold Bowtie Nanoantennas" Phys. Rev. Lett. 94, 017402 (2005).

[4] D. P. Fromm, A. Sundaramurthy, P. J. Schuck,. G. Kino and W. E. Moerner, "Cap-Dependent Optical Coupling of Single \$̧Bowtie Nanoantennas Resonant in the Visible" Nano Lett. 4, 957-961 (2004).

[5] F. Hao, C. L. Nehl, J. H. Hafner, and P. Nordlander, "Plasmon Resonances of a Cold Nanostar" Nano Lett. 7, 729 (2007).

[6] H. Wang, D. Brandl, F. Le, P. Nordlander and N. J. Halas, "NanoRice: a hybrid nanostructure" Nano Lett. 6, 827 (2006).

[7] N. F. van Hulst, "Light in chains" Nature 448, 141 (2007).

[8] K. Li, M I. Stockman, and D. J. Bergman, "Self-Similar Chain of Metal Nanospheres as an Efficient Nanolens" Phys. Rev. Lett. 91, 227402-1 (2003).

[9] T. Kalkbrenner, U. Hakanson, A. Schädle, S. Burger, C. Henkel and V. Sandoghdar, "Optical Microscopy via Spectral Modifications of a Nanoantenna" Phys. Rev. Lett. 95, 200801-1 (2005).

[10] P. Anger, P. Bharadwaj and L. Novotny, "Enhancement and Quenching of Single-Molecule Fluorescence" Phys. Rev. Lett. 96, 113002-1 (2006).
[11] T. H. Taminiau, R. J. Moerland, F. B. Segerink, L. Kuipers and N. $F$. van Hulst, " $\lambda / 4$ Resonance of an Optical Monopole Antenna Probed by Single Molecule Fluorescence" Nano Lett. 7, 28-33 (2007).

[12] R. Carminati, J.-J. Greffet, C. Henkel and J. M. Vigoureux, “Radiative and non-radiative decay of a single molecule close to a metallic nanoparticle" Opt. Commun. 261, 368Ú375 (2006).

[13] F. Tam, G. P. Goodrich, B. R. Johnson, and N. J. Halas, "Plasmonic Enhancement of Molecular Fluorescence" Nano Lett. 7, 496 (2007).

[14] J. P. Kottmann, O. J. F. Martin, D. R. Smith and S. Schultz, “Nonregularly shaped plasmon resonant nanoparticles as loclized light sources for near-field microscopy" J. Microscopy 200, 60 (2001).

[15] J. N. Farahani, D. W. Pohl, H.-J. Eisler and B. Hecht, "Single Quantum Dot Coupled to a Scanning Optical Antenna: A Tunable Superemitter" Phys. Rev. Lett. 95, 017402 (2005).

[16] E. Cubukcu, E. A. Kort, K. B. Crozier and F. Capasso, "Plasmonic laser antenna" Appl. Phys. Lett. 89, 093120 (2006).

[17] D. R. Matthews, K. Njoh, S. Chappell, R. Errington, P. Smith and H. D. Summers, "Optical antenna arrays in the visible range" Opt. Express 15, 3478 (2007).

[18] M. Paulus, P. Gay-Balmaz and 0. J. F. Martin, "Accurate and efficient computation of the Green's tensor for stratified media" Phys. Rev. E 62, 5797 (2000).

[19] M. Paulus and 0. J. F. Martin, "Light propagation and scattering in stratified media: a Green's tensor approach" J. Opt. Soc. Am. A 18, 854 (2001).

[20] 0. J. F. Martin and N. B. Piller, "Electromagnetic scattering in polarizable backgrounds" Phys. Rev. E 58, 3909 (1998).

[21] P. B. Johnson and R. W. Christy, "Optical Constants of the Noble Metals" Phys. Rev. B 6, 4370 (1972).

[22] J. D. Jackson, Classical Electrodynamics (John Wiley and Sons, inc., USA, 1999). 